\title{
Pre-expanded \\ Perforator Flaps
}

Editors

LEE L.Q. PU

CHUNMEI WANG

CLINICS IN

PLASTIC SURGERY

www.plasticsurgery.theclinics.com

January 2017 • Volume 44 • Number 1 